\title{
Developing an Iterative Tracking Algorithm to Guide a Catheter Towards Atrial Fibrillation Rotor Sources in Simulated Fibrotic Tissue
}

\author{
Prasanth Ganesan $^{1}$, Hussein Zilouchian ${ }^{1}$, Elizabeth M Cherry ${ }^{2}$, Arkady M Pertsov ${ }^{3}$, Behnaz \\ Ghoraani $^{1}$ \\ ${ }^{1}$ Department of Electrical Engineering, Florida Atlantic University, FL USA \\ ${ }^{2}$ School of Mathematical Sciences, Rochester Institute of Technology, NY USA \\ ${ }^{3}$ Department of Pharmocology, Upstate Medical University, NY USA
}

\begin{abstract}
Locating atrial fibrillation $(A F)$ rotor sources can help target ablation therapy for AF. Our aim was to develop a catheter-tracking algorithm to locate AF rotor sources using a conventional 20-electrode circular catheter. We simulated rotor-driven arrhythmias in homogeneous and fibrotic human atrial tissue and evaluated the algorithm for different initial catheter positions. The algorithm guided and detected a rotor with a success rate of greater than $97.9 \%$ independently of the initial position of the catheter with an accuracy of greater than $2.3 \pm 1.4 \mathrm{~mm}$.
\end{abstract}

\section{Introduction}

Atrial fibrillation (AF) affects more than 2.7 million people in the US alone and is the most common cardiac arrhythmia, accounting for frequent hospitalizations and increased risks of stroke, heart failure and mortality [1]. Catheter ablation therapy, the most commonly used treatment for AF, is used to electrically isolate the pulmonary veins (PVs) from the left atrium and block the ectopic signals originating from PVs to trigger the arrhythmia. The procedure has a suboptimal $40-60 \%$ success rate, which is believed to be due to the formation of additional AF sources in the atria, especially in patients with persistent $\mathrm{AF}$ [2,3].

Previous studies were performed on the design of computational mapping systems for improved detection of AF drivers outside the PVs. For the most part, this work has focused on developing 3D electroanatomic mapping based on the electrogram characteristics from a single or multipole diagnostic catheter to locate AF sources. We have developed a fundamentally different approach that, without the need to map the entire atria, utilizes bipolar electrogram recordings obtained from a conventional circular catheter to iteratively guide the catheter towards the wave source and stop when a rotor source is detected [9]. In this paper, we performed a series of simulation experiments to validate the success of this algorithm to locate a rotor in a simulated human tissue. Additionally, we compared the performance of this algorithm to that of a phase-mapping method that is similar to the recently developed Focal Impulse Rotor Modulation (FIRM) mapping (Abbott Electrophysiology), which employs a customized basket catheter to locate a rotor in the atria.

\section{Materials and Methods}

In order to evaluate our catheter-guidance algorithm, we created simulations of rotors, and generated electrograms as described below.

\subsection{Rotor Simulation}

We employed a computer simulation to generate a $10 \mathrm{~cm}$ $\mathrm{x} 10 \mathrm{~cm} 2 \mathrm{D}$ atrial tissue with a spatial resolution of 0.025 $\mathrm{cm}$ and time steps of 0.05 milliseconds. In these simulations, we used the Nygren et al. human atrial cell model [4], which was based on average voltage-clamp data recorded from the isolated single myocytes from typical bulk atrial tissue. To model fibrotic tissue, thick collagenous septa were introduced by removing electrical connections between neighboring myocytes oriented along an assumed fiber orientation (horizontal) and uniformly distributed throughout the tissue. The septa lengths varied according to a Poisson distribution with an average length of $2.5 \mathrm{~mm}$ and affected a moderate amount of the tissue $(20 \%)$. A single rotor was initiated in the tissue with homogeneous and fibrotic conduction properties [10] (see in Figure 1). As expected, the addition of septa (heterogeneity) altered the wave propagation dynamics making the wave slower in the vertical direction and faster in the horizontal. Rotors were initiated by a cross-field stimulation protocol. 


\subsection{Catheter Simulation}

Two types of catheters were simulated: 20-pole Lasso and atrial basket catheters. A 20-pole Lasso catheter (Biosense Webster) with $15 \mathrm{~mm}$ diameter and 4.5-1-4.5 mm electrode spacing was simulated (Figure 1). First, unipolar electrograms were simulated by following Ref. [11]. Next, ten bipolar electrograms were calculated from the pairs of the 20 unipolar electrograms by subtracting the unipolar electrograms. These bipolar electrograms were further used in the catheter-guidance algorithm.

A FirMap (Abbott Electrophysiology) atrial basket catheter with the highest available resolution (i.e., diameter of $40 \mathrm{~mm}$ and electrode spacing of $6.8 \mathrm{~mm}$ ) was simulated on the 2D tissue. Figure 1 shows the electrode spacing of the basket catheter placed horizontally (splines in the horizontal direction). The unipolar electrograms were obtained at the electrode locations [11]. These unipolar electrograms were used by the phase mapping method to locate rotors.

\subsection{Catheter Guidance Method}

We developed a catheter-guidance algorithm that starts by placing the catheter at an arbitrary location on the atrial tissue and uses the patterns of the 10 bipolar electrograms of an circular catheter (here, a Lasso catheter) to gradually guide the catheter towards a rotor and detect when a rotor is located [5, 9]. The proposed catheter-guidance method consists of three main parts.

Bipolar Electrogram Characteristics: At each catheter placement, the temporal activations of each bipole are tagged to determine the cycle length (CL), first activated bipole (FAB), and rotor propagation ratio (RPR). The bipolar characteristics for an arbitrary Lasso location are indicated in Figure 1 C. CL is calculated as the average of the time delays of two sequential activations through all bipoles. The FAB is determined as the first bipole that encounters the wavefront. RPR is calculated as the ratio of the sum of the conduction delays (CD) between activations of adjacent bipoles in one cycle to CL.

Catheter Guidance: The algorithm starts with an initial placement of a Lasso on the atrial tissue. The information about the location and the type of source are hidden from the algorithm and only the electrogram data are known. At any catheter placement, the electrograms are recorded and information about the CL, FAB, and RPR is extracted. The algorithm then guides the center of the Lasso to the location of the FAB while the rotor source condition (described below) is checked at every location. This process is repeated until an AF source is identified or when the algorithm failed to detect the source within 25 iterations.

Rotor Detection: The RPR value at catheter placement is close to 1 when the circular catheter encompasses a ro- tor. In this study, a rotor is detected if $R P R \geq 0.6$, in order to accommodate for rotor meandering and complex fractionated electrograms caused by the collagenous septa in fibrotic tissue simulations. The algorithm detects a rotor when two close locations (within $1 \mathrm{~cm}$ ) with $\mathrm{RPR} \geq 0.6$ are identified.

\subsection{Phase Mapping Method}

The phase-mapping method uses unipolar electrograms obtained from the basket catheter to identify the phase at the recording location at any instant of time and detect the location of phase singularities as a rotor source.

Generating Phase Maps: The unipolar electrograms at any instant are used to generate the instantaneous phase maps of all the electrodes by a phase value between $-\pi$ and $\pi$. First, the extracellular voltage was spatially interpolated using bilinear interpolation to achieve a grid spacing of at least $4 \mathrm{~mm}$ in all directions. In this paper, we implemented Kuklik et al.'s phase mapping algorithm [6]. The method starts by reconstructing a unipolar signal using cascaded sinusoidal wavelets with wavelength based on the cycle length of the unipolar signal. Next, the instantaneous phase is determined using the Hilbert transform of the reconstructed wave.

Rotor Detection: The location on the phase map where all the phases merge is known as the point of singularity and represents the location of the rotor center. To detect these phase singularity points, we use a well-known algorithm based on the Iyer-Gray method [7]. The method finds the pairwise phase differences in eight nearest neighbors of any point. If any of these difference values is less than or equal to $\pi$ then the value remains unchanged, but if the value is positive and greater than $\pi$, then $2 \pi$ is subtracted from it. In all other cases, $2 \pi$ is added to the value. The location(s) on the phase map that produces the maximum phase difference value (i.e., absolute phase difference closest to $2 \pi$ ) is determined as the phase singularity point. A phase singularity that lasts at least one cycle length of the arrhythmia is detected as the location of a rotor.

\section{Results and Discussions}

We performed a series of experiments to investigate the performance of the proposed catheter-guidance algorithm that uses a commonly used circular catheter to locate rotors, and also compared it with a common phase-mapping method that is based on customized basket catheters.

Catheter-Guidance Method Using a Lasso Catheter: The algorithm started by placing a Lasso catheter on an arbitrary location on the atrial tissue and gradually guided the catheter based on the electrogram characteristics as described in the Methods section. The algorithm stopped the 

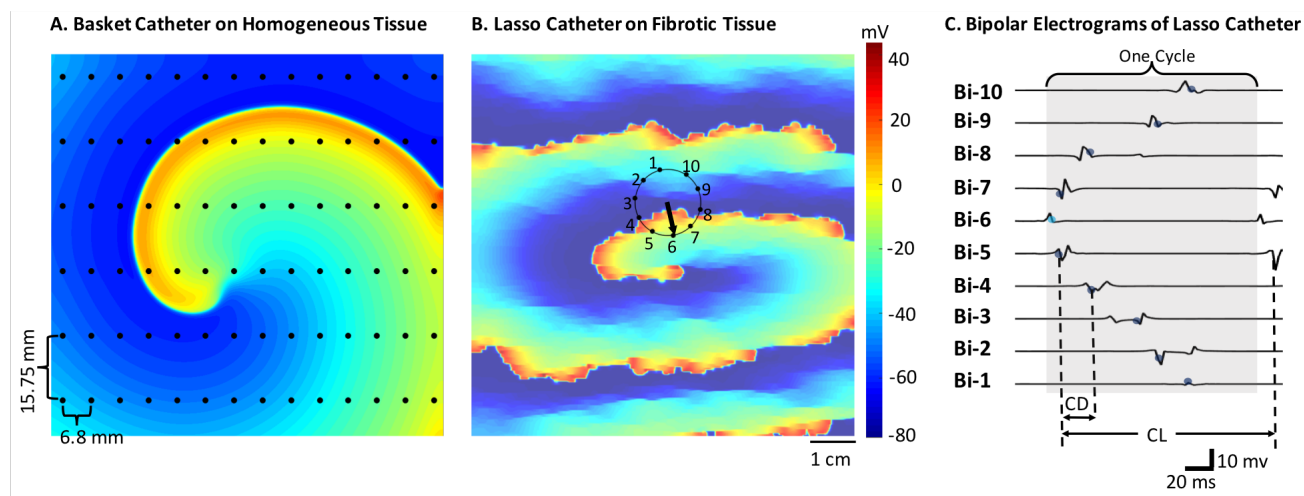

Figure 1. A. Simulated rotor in homogeneous atrial tissue. Black dots show the mapping of the individual electrodes of a 64-electrode basket catheter onto the atrial surface. B. Simulated rotor in fibrotic tissue. Black circle shows the outline of the Lasso catheter. Black dots show the centers of bipoles. C. Simulated electrograms at the Lasso catheter position in B.

guidance when a rotor was detected. The guidance paths for two different starting locations are shown in Figure $2 \mathrm{~A}$. We repeated the experiment by guiding the catheter on the fibrotic tissue from the same starting locations (Figure 2B). As expected, the guidance path has changed from the homogeneous AF simulation; however, the developed catheter-guidance algorithm successfully guided the catheter towards the rotor center in both simulations and stopped within a catheter radius distance from the center of the rotor.
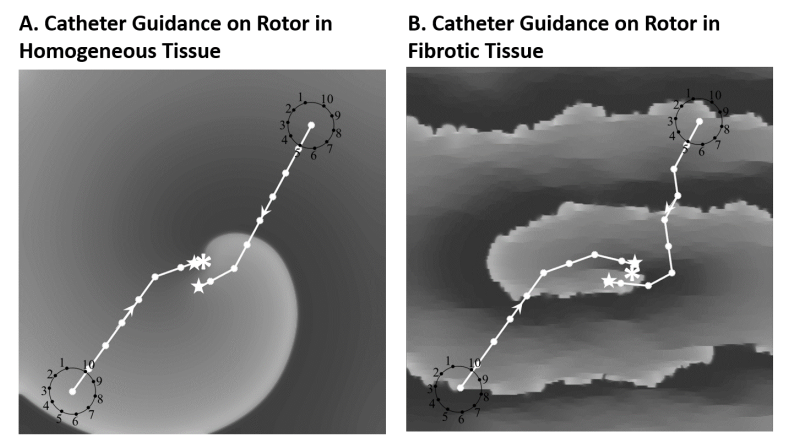

Figure 2. Catheter guidance trajectories in homogeneous (A) and fibrotic (B) tissue. White dots indicate the sequential position of the center of the catheter. Asterisk shows the actual center of the rotor. Stars indicate the rotor positions detected by the algorithm for two different initial placements of the catheter.

We tested the algorithm for locating the AF rotor source for 114,921 different initial catheter positions. A rotor detection is considered a successful detection if the detected location is within $4 \mathrm{~mm}$ (an ablation catheter tip diameter) of the average trajectory of the rotor tip [12]. It is considered a false detection if the detected rotor is more than $4 \mathrm{~mm}$ from the average trajectory of the rotor tip. A test is considered a detection failure if the algorithm is not able to detect a rotor location (in fewer than 25 catheter placements in case the of the catheter guidance algorithm).

The algorithm located the rotor in the homogeneous tissue for $100 \%$ of trials within $2.3 \pm 1.4 \mathrm{~mm}$ of the rotor center with an average $7 \pm 2$ Lasso placements. We repeated this experiment on the fibrotic tissue. The average distance of the detected rotors was $3.0 \pm 1.3 \mathrm{~mm}$ from the rotor location. The algorithm was successful in $97.9 \%$ of the trials and successfully located the rotor within $1.14 \pm 1.06$ $\mathrm{mm}$ of the rotor center with an average $8 \pm 3$ Lasso placements. The average distance of the false detected rotors was $4.44 \pm 0.24 \mathrm{~mm}$ from the rotor indicating that they were not too far from the rotor tip.

Phase-Mapping Method Using a Basket Catheter: The basket catheter was placed on the homogeneous and fibrotic tissue as shown in Figure 1 The phase mapping method was used to locate the rotor. The derived phase map is shown in Figure 3. The detected rotor center (i.e., phase singularity) is shown in black asterisks in this figure. In the homogeneous case, the phase singularity point was stable at one single location at $100 \%$ of the times. However, in the fibrotic case, there were several phase singularities indicating the presence of several rotors. Figure 3D shows the phase singularities that lasted more than one cycle length of the arrhythmia and were detected as rotor locations. As shown in this figure, the phase-mapping method using a basket catheter was able to detect the true location of the rotor in case of the homogeneous tissue. However, the basket catheter did not provide sufficient spatial resolution for the phase-mapping method to successfully locate the rotor in the case of fibrotic tissue. In fact, no correct rotor was detected in this case. This observation was in agreement with the previous reports [12] that the low-resolution basket catheters were prone to false detections. The locations of the detected rotors were compared to the location of the rotor trajectory. The Euclidean dis- 
tance between each location and the true rotor location was calculated for each simulation, and the mean distance was computed for the homogeneous and fibrotic tissue cases as $1.5 \pm 0.6 \mathrm{~mm}$ and $36.4 \pm 11.1$, respectively.

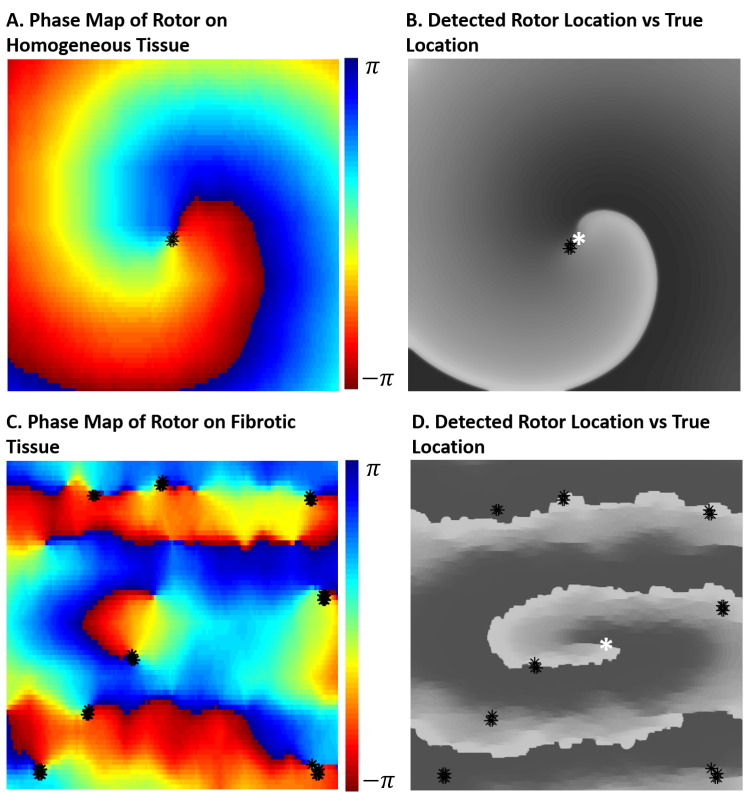

Figure 3. Performance of the phase-mapping method in homogeneous (A,B) and fibrotic (C,D) tissues - A. Phase map of a rotor in homogeneous tissue. The phase singularity is indicated by a black asterisk. B. The voltage map (in gray scale) of the rotor in A. The white asterisk indicates the true location of the rotor. C. Phase map and the phase singularities produced by a single rotor in fibrotic tissue (the corresponding voltage map is shown in Panel D). D. Voltage map (in gray scale) of the rotor shown in Panel C with superimposed phase singularities (black asterisks).

\section{Conclusion}

We developed a novel algorithm to guide a multi-polar, circular diagnostic catheter towards a rotor center and detect the location of the rotor. Our experiments demonstrated that the algorithm was successful in both homogeneous and fibrotic rotor cases compared to methods based on a 64-electrode basket catheter. The developed algorithm could be used as add-on software in existing electrophysiology laboratory systems to help locate patient-specific AF ablation targets outside the pulmonary veins. Such a system may significantly improve the success of AF ablation and facilitate treating patients. In the future, we plan to extend the assessment of the developed algorithm to various rotor-driven atrial arrhythmias, such as patchy myocardial scars and complex fibrillatory activation patterns.

\section{Acknowledgements}

This work was supported by the National Institutes of Health, R15HL127663 grant.

\section{References}

[1] Mozaffarian D, et al. Heart disease and stroke statistics 2015 update a report from the American Heart Association. Circulation, 2015; 131(4): e29-e322.

[2] Narayan S M, et al. Treatment of atrial fibrillation by the ablation of localized sources: CONFIRM (Conventional Ablation for Atrial Fibrillation with or Without Focal Impulse and Rotor Modulation) trial. JACC, 2012; 60(7): 628-636.

[3] Ghoraani B, et al. Localized rotational activation in the left atrium during human atrial fibrillation: Relationship to complex fractionated atrial electrograms and low-voltage zones. Heart Rhythm, 2013; 10(12): 1830-1838.

[4] Nygren A, et al. Mathematical model of an adult human atrial cell the role of $\mathrm{k}+$ currents in repolarization. Circulation Research, 1998; 82(1):63-81.

[5] Ganesan P, et al. Characterization of electrograms from multipolar diagnostic catheters during atrial fibrillation. BioMed research international, 2015.

[6] Kuklik P, et al. Reconstruction of instantaneous phase of unipolar atrial contact electrogram using a concept of sinusoidal recomposition and hilbert transform. IEEE Transactions on Biomedical Engineering, 2015; 62(1): 296-302.

[7] Iyer A N, Gray R A. An experimentalists approach to accurate localization of phase singularities during reentry. Annals of Biomedical Engineering, 2001; 29(1):47-59.

[8] Ganesan P, et al. Development of a novel probabilistic algorithm for localization of rotors during atrial fibrillation. IEEE Annual International Conference of the Engineering in Medicine and Biology Society, 2016; 493-496.

[9] Salmin A, et al. A novel catheter-guidance algorithm for localization of atrial fibrillation rotor and focal sources. IEEE Annual International Conference of the Engineering in Medicine and Biology Society, 2016; 501-504.

[10] Ganesan P, et al. Simulation of Spiral Waves and Point Sources in Atrial Fibrillation with Application to Rotor Localization. IEEE International Symposium on ComputerBased Medical Systems, 2017; 379-384.

[11] Bueno-Orovio A, Cherry E M , and Fenton F H. Minimal model for human ventricular action potentials in tissue. Journal of Theoretical Biology, 2008; 253(3):544-560.

[12] Roney C, Cantwell C, et al. Spatial resolution requirements for accurate identification of drivers of atrial fibrillation. Circ Arrhythm Electrophysiol, 2017; 10(5):e004899

Address for correspondence:

Name: Behnaz Ghoraani

Full postal address: College of Engineering \& Computer Science, Engineering East, Bldg. 96, 777 Glades Rd, Boca Raton, FL 33431 E-mail address: bghoraani@ieee.org 